Classification

Physics Abstracts

$05.20-05.50-64.60-64.70$

\title{
A majority-rule model : real-space renormalization-group solution and finite size scaling
}

\author{
C. Tsallis \\ Centro Brasileiro de Pesquisas Físicas/CNPq, Av. Wenceslau Braz, 71, fundos \\ 22290 Rio de Janeiro, RJ-Brazil
}

(Reçu le 30 mars 1982, révisé le 11 mai, accepté le 13 mai 1982)

\begin{abstract}
Résumé. - A l'aide d'une règle de majorité simple, nous formulons un modèle statistique géométrique $d$-dimensionnel ( $d$ peut être fractionnaire); ce modèle présente, en fonction d'une certaine probabilité $p$ d'occupation indépendante, une transition de phase continue. Nous calculons exactement, dans le cadre d'un groupe de renormalisation dans l'espace réel ( $b$ étant le facteur d'échelle linéaire), le point critique $p_{\mathrm{c}}$ et l'exposant $v$ de longueur de corrélation. L'hypothèse bien connue d'échelle de taille finie $v(b)-v \propto 1 / \ln b$ (dans la limite $b \rightarrow \infty$ ) est montrée analytiquement sur un exemple non trivial probablement pour la première fois. Nous suggérons aussi une nouvelle procédure d'extrapolation de tailles finies dont la convergence est plus rapide.
\end{abstract}

\begin{abstract}
Through a simple majority-rule a statistical geometrical $d$-dimensional model ( $d$ can even be a fractal dimensionality) is formulated which presents a continuous phase transition as a function of a certain independent occupancy probability $p$. Both critical point $p_{\mathrm{c}}$ and $/$ correlation length " exponent $v$ are exactly calculated through real-space renormalization-group (with linear scaling factor $b$ ). The well-known finite size scaling hypothesis $v(b)-v \propto 1 / \ln b$ (in the limit $b \rightarrow \infty$ ) is analytically exhibited. A more rapidly convergent finite size extrapolation procedure is presented.
\end{abstract}

The simple majority-rule (see [1]) has been frequently used during recent years within realspace renormalization-group ( $R G)$ approximate treatments of several thermal models (mainly Ising-like). We shall use it herein as the basis for constructing a geometrical model which presents a continuous phase transition. Let us consider a macroscopic square checkerboard (yet uncoloured) which is going to be randomly and independently (quenched model) occupied by black and white plaquettes (whose respective occupancy probabilities are $p$ and $(1-p)$ ). We arbitrarily choose an elementary square (hereafter referred to as the centre) and assume, for convenience, that it is always black (this weak restriction in the ensemble of possible macroscopic occupancy configurations has clearly no relevance in the thermodynamic limit). For a given macroscopic configuration associated to $p<1 / 2$ we proceed as follows : by starting from the black centre (which corresponds to a degree of consultation $n=0$ ) we check if the inclusion of its immediate neighbourhood (which corresponds to $n=1$; the subsystem under consideration contains now $N_{1}$ elementary squares; $N_{1}=9$ in figure $1 a$ ) preserves the black majority; if this is the case we expand even more our subsystem $\left(n=2\right.$; the new subsystem contains $N_{2}$ elements; $N_{2}=25$ in 
figure 1a); we keep on through this procedure up to the point where the black majority is reversed (the reversal point is characterized by a consultation degree $n_{\mathrm{r}} ; n_{\mathrm{r}}=2$ in figure $1 a$ ). When this change occurs (and necessarily occurs as we have assumed $p<1 / 2$ ) we consider a new random configuration (still associated to the same value of $p$ ) and go through the same operational sequence, thus obtaining a new value for $n_{\mathrm{r}}$; we note $\xi$ the (arithmetic) mean value of the $\left\{n_{\mathrm{r}}\right\}$ associated with a thermodynamically great number of configurations; clearly $\xi$ diverges (presumably as $\left(p_{\mathrm{c}}-p\right)^{-\mathrm{v}}$ with $\left.p_{\mathrm{c}}=1 / 2\right)$ when $p$ approaches $p_{\mathrm{c}}$. If $p>1 / 2$ the procedure to be followed is exactly the same excepting for the fact that only configurations leading to finite $n_{\mathrm{r}}$ enter into the calculation of $\xi$, which is now expected to diverge as $\left(p-p_{\mathrm{c}}\right)^{-v}$ in the vicinity of $p_{\mathrm{c}}$.

(a)

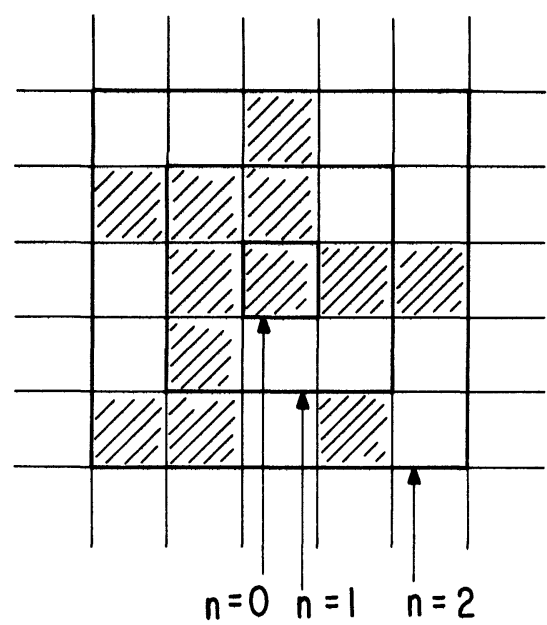

(b)

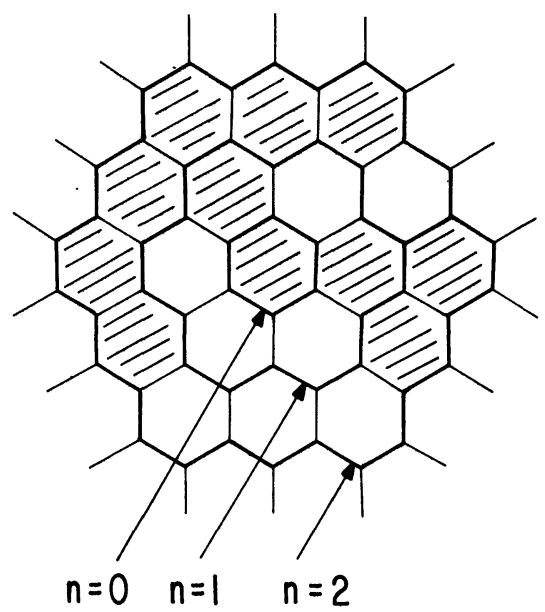

Fig. 1. - Examples of occupancy configurations. $(a) N_{n}=(2 n+1)^{2}$ and $n_{\mathrm{r}}=2 ;(b) N_{n}=3 n(n+1)+1$ and $n_{\mathrm{r}}=1$.

Now that the majority model (MM) has been introduced we intend to calculate $v$ within a RG framework which renormalizes finite squares (with side length $b$ ) into smaller ones (side length $b^{\prime}<b$ ); both $b$ and $b^{\prime}$ are odd numbers in order to avoid majority ambiguities. For analytical simplicity we shall work, in the RG framework, with no colour restriction on the centre of the squares (if we impose the black colour for the centres, the final asymptotic results in the $b \rightarrow \infty$ region are exactly the same). Let us first consider the simplest case, i.e. $b=3$ and $b^{\prime}=1$. The RG recursion is given by

$$
p^{\prime}=R_{3}(p) \equiv p^{9}+9 p^{8}(1-p)+36 p^{7}(1-p)^{2}+84 p^{6}(1-p)^{3}+126 p^{5}(1-p)^{4}
$$

which presents (besides the two trivial stable fixed points $p=0$ and $p=1$ ) an unstable fixed point at $p=1 / 2$ (exact answer) and leads to $\lambda_{3} \equiv \mathrm{d} R_{3}(p) /\left.\mathrm{d} p\right|_{p=1 / 2}=315 / 2^{7}$, therefore to the approximate critical exponent $v_{3,1}=\ln 3 / \ln \lambda_{3} \simeq 1.2199$. For general values of $\left(b, b^{\prime}\right)$ and considering now a d-dimensional hypercubic checkerboard, equation (1) becomes

with

$$
R_{b^{\prime}}\left(p^{\prime}\right)=R_{b}(p) \quad\left(b=3,5,7, \ldots ; b^{\prime}=1,3, \ldots, b-2\right)
$$

$$
R_{b}(p) \equiv \sum_{i=0}^{\left(b^{d}-1\right) / 2}\left(\begin{array}{c}
b^{d} \\
i
\end{array}\right) p^{b^{d}-i}(1-p)^{i} .
$$


This recursion admits, for all $d$ and $\left(b, b^{\prime}\right)$, the unstable fixed point $p=1 / 2$ (exact answer) and leads to

with

$$
v_{b, b^{\prime}}=\frac{\ln \left(b / b^{\prime}\right)}{\ln \left(\lambda_{b} / \lambda_{b^{\prime}}\right)}
$$

$$
\left.\lambda_{b} \equiv \frac{\mathrm{d} R_{b}(p)}{\mathrm{d} p}\right|_{p=1 / 2}=\frac{b^{d}}{2^{b^{d}-1}}\left(\begin{array}{l}
b^{d}-1 \\
\frac{b^{d}-1}{2}
\end{array}\right) \quad(b=1,3,5, \ldots) .
$$

By using Stirling's approximation $\left(\ln N !=N \ln N-N+\frac{1}{2} \ln (2 \pi N)+\frac{1}{12 N}+\mathrm{o}\left(1 / N^{3}\right)\right.$ in the limit $N \rightarrow \infty$ ) we straightforwardly obtain (in the limit $b \rightarrow \infty$ )

$$
v_{b, 1}=\frac{2 / d}{1-\frac{\ln (\pi / 2)}{d \ln b}}+o\left(1 / b^{d} \ln b\right)
$$

and

$$
v_{b, b-2}=\frac{2 / d}{1-\frac{1}{2 b^{d}}}+\sup \left[\mathrm{o}\left(1 / b^{d+1}\right), \mathrm{o}\left(1 / b^{2 d}\right)\right]
$$

and finally

$$
v=\lim _{\substack{b \rightarrow \infty \\ b^{\prime}<b}} v_{b, b^{\prime}}=\frac{2}{d}
$$

This result deserves a few comments. First of all, how is it we obtain a phase transition for any dimensionality, even for $d=1$ ?; this is clear once we realize that the majority-rule implies long range (as a matter of fact, infinite range) interactions as, at any given degree of consultation $n$, even very distant elements enter into consideration on equal standing as the neighbouring ones; for this same reason we do not expect that a crossover towards classical values for the critical exponents (e.g. $v$ ) exists for any finite dimensionality (i.e. the upper critical dimensionality above which a mean field type theory is valid diverges). Our second comment refers to standard site percolation to which the MM might, at first sight, look isomorphic; this is clearly wrong as the question " can our region be spanned through (let us say first-neighbouring) black plaquettes ?" is very different from " in our region, are the black plaquettes majority ? " (e.g. in the subsystem $n=2$ of figure $1 a$, the answer to the first question is yes, where it is no to the second one; the opposite answers are possible as well). The MM might also look like similar to the Ising one : although both binary, these models are quite different as, at any finite temperature, neighbouring plaquettes are likely of the same (different) "colour» in the ferromagnetic (antiferromagnetic) Ising model whereas there is no such correlation in the MM. We might also think about more sophisticated models, like the triangular lattice quenched bond-mixed Ising one with a distribution law for the coupling constant $J$ given by $P(J)=(1-x) \delta\left(J-J_{0}\right)+x \delta\left(J+J_{0}\right)$ with $J_{0}>0$; if we consider an elementary triangular plaquette and associate " black " to " frustated " we obtain $p=x^{3}+3(1-x)^{2} x(x=0,1 / 2,1$ respectively imply $p=0,1 / 2,1)$, but we can easily verify that the probability for two first-neighbouring plaquettes being « frustrated » does not equal $p^{2}$ as in the MM. Our last comment concerns a possible generalization of the present results. There is clearly no reason for the consultation sequence $N_{n}$ to grow (while the consultation degree $n$ runs over all natural numbers) as that of an hypercubic lattice (i.e. $\left.N_{n}=(2 n+1)^{d}\right)$ and 
any regular lattice can be used as well (see Fig. $1 b$ ); furthermore $N_{n}$ has not to be related to any regular (or even irregular) lattice : it can be an arbitrary one. In that case we can define a fractal dimensionality (see [2]) $d_{\mathrm{f}} \equiv \lim _{n \rightarrow \infty} \frac{\ln N_{n}}{\ln (2 n+1)}$ (it is clear that all $d$-dimensional regular lattices lead to $\left.d_{\mathrm{f}}=d\right)$; for this MM we expect $p_{\mathrm{c}}=1 / 2$ and $v=2 / d_{\mathrm{f}}$.

Let us now turn back to equations (4) and (5) (and the asymptotic behaviours (6) and (7)) in order to discuss the well-known finite size scaling hypothesis [3] frequently used in pure [4] and RG [5] Monte-Carlo treatments of statistical models, and herein analytically exhibited. The critical value $p_{c}=1 / 2$ is exactly obtained within the present $\mathrm{RG}$ for all $\left(b, b^{\prime}\right)$, therefore we confine our discussion onto the behaviour of $v_{b, b}$, for large values of $b$. We shall make three remarks :

i) It is systematically assumed in the RG Monte-Carlo treatments of statistical models that $\left(v_{b, 1}-v\right)$ (therefore $\left.\left(1 / v_{b, 1}-1 / v\right)\right)$ is proportional to $1 / \ln b$ in the limit $b \rightarrow \infty$ : equation (6) confirms this assumption which presumably for most models is still true for $\left(v_{b, b^{\prime}}-v\right)$ with any fixed value of $b^{\prime}$ (or values of $b^{\prime}$ growing, with $b$, in a sufficiently slow manner);

ii) As seen from equation (6) the asymptotic regime is more rapidly attained by $1 / v_{b, 1}$ than by $v_{b, 1}$; consequently, for practical purposes, extrapolations $1 / v_{b, 1} v s .1 / \ln b$ are expected to provide

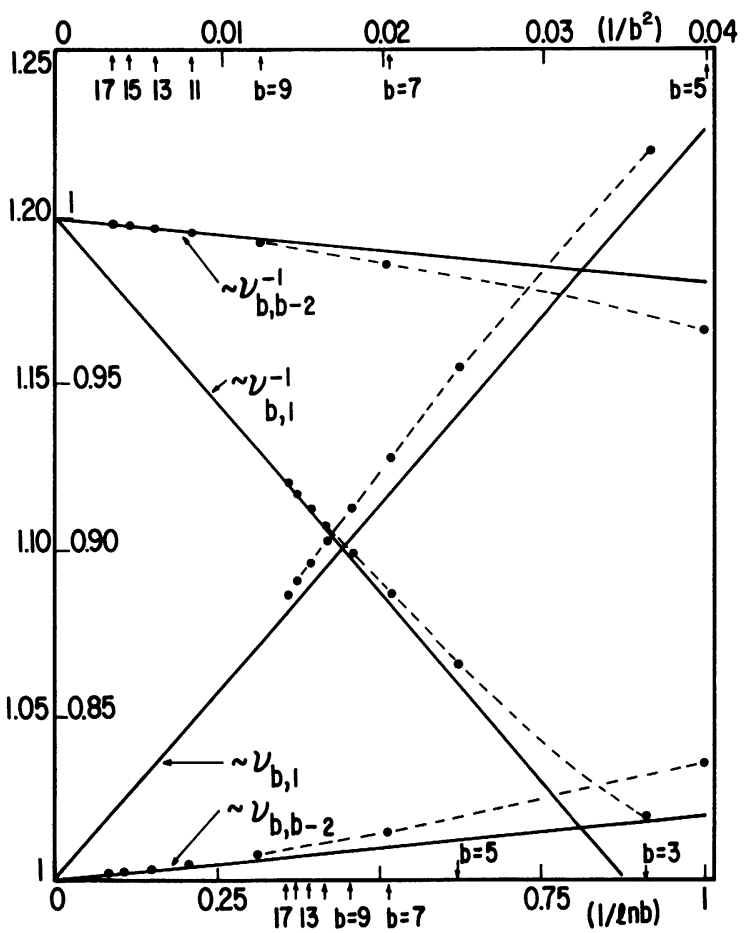

Fig. 2. - Critical exponent $v$ finite size scaling exhibited for the $d=2 \mathrm{MM}$. Full lines represent the $b \rightarrow \infty$ asymptotic behaviours; dashed lines are guides to the eye and run over the actual approximate values of $v$ (represented by dots). The ordinate scale for the $v$-lines $\left(v^{-1}\right.$-lines) is the outside (inside) one; the abscissa scale for the $v_{b, 1^{-}}$and $v_{b, 1^{-1}}^{-1}$-lines ( $v_{b, b-2^{-}}$and $v_{b, b-2^{-1}}^{-1}$ lines) is the bottom (top) one. Concerning numerical procedures remark that : (a) the standard $v_{b, 1}^{-1} v s .1 / \ln b$ one is superior to the standard $v_{b, 1} v s .1 / \ln b$ one; (b) both $v_{b, b-2}$ vs. $b^{-d}$ and $v_{b, b-2}^{-1} v s . b^{-d}$ (numerically slightly better) procedures are superior to the standard ones (using 1/ln $b$ ). A numerical illustration : $v_{13,1}=1.09583, v_{15,1}=1.09047, v_{13,11}=1.00353$ and $v_{15,13}=1.00258$; the two points linear extrapolation of these values provide the following errors for $v:-0.56 \%$ in the case $v_{b, 1} v s .1 / \ln b, 0.26 \%$ in the case $1 / v_{b, 1} v s .1 / \ln b,-0.027 \%$ in the case $v_{b, b-2} v s .1 / b^{2}$ and $-0.026 \%$ in the case $1 / v_{b, b-2}$ vs. $1 / b^{2}$. 
higher numerical precision than that obtained in extrapolations $v_{b, 1} v s .1 / \ln b$; no such $a$ priori expectation exists related to equation (7), therefore a $v_{b, b-2} v s$. $1 / b^{d}$ extrapolation can lead to results practically as good as those obtained through a $1 / v_{b, b-2} v s .1 / b^{d}$ extrapolation (the MM $d=2$ case is illustrated in figure 2 ).

iii) As seen from equations (6) and (7) the asymptotic regime is more rapidly attained if the sequence $(b, b-2)$, rather than the $(b, 1)$ one, is used (the $\mathbf{M M} d=2$ case is illustrated in figure 2$)$; in more general terms, sequences $\left(b, b^{\prime}\right)$ such that $b / b^{\prime} \rightarrow 1$ are, for a possibly very large class of extrapolations, to be a priori preferred to those which imply $b / b^{\prime} \rightarrow \infty$.

To synthetize let us say that the present work suggests, for the numerical calculation of the " correlation length " critical exponent $v$ associated to a possibly large variety of statistical systems, the use of extrapolations $1 / v_{b, b^{\prime}} v s .1 / b^{d}$ with $b^{\prime}$ running as close to $b$ as possible, rather than the standard ones (namely $v_{b, 1} v s$. $1 / \ln b$ or $1 / v_{b, 1} v s$. $1 / \ln b$ ). This type of advantage has already been analytically observed [6] on a phenomenological renormalization approach for the $d=1$ Ising model ; the fact that the range of the forces seems to be irrelevant reinforces the expectation that these behaviours are quite general. If we focus the majority model (which admits, in fact, several natural extensions on which we are presently working) introduced herein, let us recall that its exact critical point and " correlation length " exponent respectively are $p_{\mathrm{c}}=1 / 2$ and $v=2 / d$ for the $d$-dimensional hypercubic lattice (for an arbitrary sequence $N_{n}$ we expect $v=2 / d_{\mathrm{f}}$ where $d_{\mathrm{f}} \equiv \lim _{n \rightarrow \infty} \frac{\ln N_{n}}{\ln n}$ is a fractal dimensionality). A straightforward application of the present majority model could clearly be within mathematical models for political sciences (see also page 432 of [1]); it should be interesting to search for physical applications within the standard thermal and/or geometrical statistical problems (see, for example, [7] and references therein). Finally the introduction, into this model, of a " magnetic field " would allow for the calculation of a second critical exponent (besides $v$ ), which would in turn enable (by assuming that the standard scaling laws hold) a first insight into quantities such as the susceptibility and the critical point $(p=1 / 2)$ correlation function.

Acknowledgments. - I am very grateful to E. V. Anda, E. M. F. Curado, R. Lobo and A. C. N. de Magalhães for relevant remarks.

\section{References}

[1] NiemeiJer, Th. and Van Leeuwen, J. M. J., "Renormalization Theory for Ising-like Spin Systems " from Phase Transitions and Critical Phenomena, ed. by C. Domb and M. S. Green, vol. 6 (Academic Press) 1976 , p. 425.

[2] Mandelbrot, B. B., Fractals : Form, Chance and Dimension (W. H. Freeman, San Francisco) 1977.

[3] FisheR, M. E., in Proceedings of the International Summer School Enrico Fermi 1970, Course 51, Varenna, Italy, ed. by M. S. Green (Academic, New York) 1971;

FISHER, M. E. and BARBer, M. N., Phys. Rev. Lett. 28 (1972) 1516;

Sur, A., Lebowitz, J. L., Marro, J., Kalos, M. L. and KirkPatrick, S., J. Stat. Phys. 15 (1976) 345 ; BRÉZIN, E., preprint (1981).

[4] Landau, D. P., Phys. Rev. B 13 (1976) 2997, 14 (1976) 255 and 22 (1980) 2450;

Hoshen, J., Stauffer, D., Bishop, G. H., Harrison, R. J. and Quinn, G. D., J. Phys. A 12 (1979) 1285.

[5] Suzuki, M., Prog. Theor. Phys. 58 (1977) 1142 ;

Reynolds, P. J., Stanley, H. E. and Klein, W., Phys. Rev. B 21 (1980) 1223 ;

Eschbach, P. D., Stauffer, D. and Herrmann, H. J., Phys. Rev. B 23 (1981) 422. 
[6] Derrida, B., De Seze, L. and Vannimenus, J., Disordered Systems and Localization (Conference Rome 1981), Lecture Notes in Physics 149, 46, ed. by C. Castellani, C. Di Castro and L. Peliti (Berlin, Springer-Verlag).

[7] Tsallis, C. and Levy, S. V. F., Phys. Rev. Lett. 47 (1981) 950 ;

Martin, H. O. and Tsallis, C., J. Phys. C 14 (1981) 5645;

Chao, N. C., Schwachheim, G. and Tsallis, C., Z. Phys. B 43 (1981) 305 ;

Curado, E. M. F., Tsallis, C., Levy, S. V. F. and de Oliveira, M. J., Phys. Rev. B 23 (1981) 1419. 